\title{
A Service-Centric Solution for Wireless Sensor Networks
}

\author{
Hua Zhou, Zhiqiu Huang, and Guoan Zhao \\ College of Information Science and Technology \\ Nanjing University of Aeronautics and Astronautics \\ Nanjing, Jiangsu 210016, China \\ Email: \{zhouhua; zqhuang; nop\}@nuaa.edu.cn
}

\begin{abstract}
With the development of the Internet of Things (IoT), the integration of wireless sensor networks (WSNs) and radio frequency identification (RFID) has attracted more and more attention. A lot of efforts have been made to connect and share the resources gathered by the IoT to meet industry requirements. In this chapter, we overview the current solution of WSNs, and extend the integration of WSNs and RFID as a service-centric solution for WSN (SWSN) combined with semantic web Services. In our proposal, both the WSN sensor nodes and the RFID tags are intelligent SWSN nodes which provide services, and industry applications are clients of such services. In order to achieve this, we provide middleware support for the SWSN and present the evaluation and implementation of the SWSN solution.
\end{abstract}

Keywords - wireless sensor network; RFID; semantic web service; middleware; industry application

\section{INTRODUCTION}

With the development of the Internet of Things (IoT), more and more attentions have paid to the services that the IoT can provide for the industry. The integration of the two important technologies: the wireless sensor network (WSN) and the radio frequency identification (RFID) has been widely used in industry and research [7]. In the past, the development of WSN and RFID are considered as distinct technologies. WSN can gather environment information using simple integrated sensors. RFID and the electronic product code (EPC) network are used for tracking the information in the global supply chain. However, the industry and academia have tried to develop an Internet-based infrastructure to link the physical identification data and its related information together to meet the application requirements of industry or research. Therefore, the integration of WSN and RFID is necessary and possible for real-world application and computing.

First, recent advances in micro-electro-mechanical system (MEMS) technology, wireless communications, and digital electronics have enabled the development of low-cost, lowpower, multifunctional sensor nodes that are small in size and communicate over short distances [9]. These tiny sensor nodes, which consist of sensing, data processing, and communicating components, leverage the idea of sensor networks based on collaborative effort of a large number of nodes.

With the help of hundreds to thousand sensor nodes in a WSN, sensors can gather the physical information and transfer the data to the base station to support the services and applications of the IoT.

Second, in the world of RFID, a EPC is assigned to a RFID tag to map the real world object into the information system to support the RFID applications of the IoT [7]. Figure 1 presents a scalable distributed application RFID locator [13] based on an EPC network.

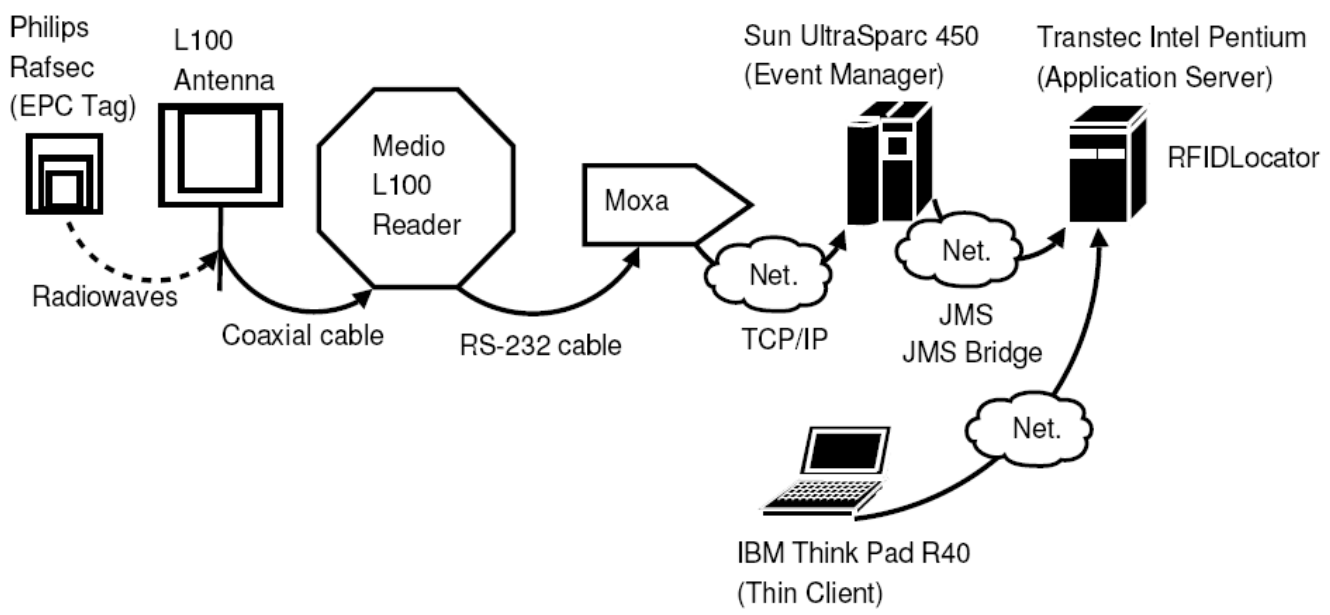

Figure 1. Components of the EPC network infrastructure. 
The extension of the RFID EPC-global architecture, the extended wireless sensor network (EWSN), can play a role as the framework for gathering, filtering and sharing both RFID and WSN data [10]. However, the different features of sensors and tags, and their networks in EWSN make the collection and analysis of data very hard because this integrated system is lack of uniform operations or a standard representation of data. Therefore, the Service-Oriented Architecture (SOA) provides a solution to the mixed RFID and WSN system. We extend the EWSN system with SOA to a service-centric wireless sensor network (SWSN), which can describe, discover services from different EWSN nodes and networks using XML and SOAP standards [1]. In this architecture, both of the RFID tags and the WSN sensor nodes are intelligent SWSN nodes. The SWSN nodes and the information gathered by the nodes are distributed reusable resources in the real world.

On the other hand, the middleware of the architecture plays an important role in collecting SWSN data form distributed nodes [4]. Therefore, the integration of WSN and RFID will provide many more advantages in real-world pervasive computing than WSN or RFID itself [7]. And the extension of the EPC architecture with SOA can provide a smooth way to integrate the RFID and WSN system for better IoT applications.

The remainder of this paper is organized as follows: In Section 2, we give background information. In Section 3, we describe the system architecture for the EWSN. In Section 4, we present the service-centric ESWSN as SWSN. In Section 5, we present the challenge in the implementation of the SWSN system. We conclude our paper in Section 6.

\section{BACKGROUND}

Wireless sensor networks (WSNs) are composed of a variety number of sensor nodes that can communicate over-theair to each using an embedded radio. [1]

Sensor nodes are placed in an area where a particular phenomenon is expected to occur. The sensor nodes sense the information of the phenomenon, and collect and rout the data to the sink sensor, which can receive, process and store the data from other nodes [2]. Figure 2 gives the architecture of the data processer through the sink sensor. Finally the data are sent to the base station, which extracts and disseminates data information from the network, and much more importantly, it is a powerful server which plays an important role in data processing and storage [8].

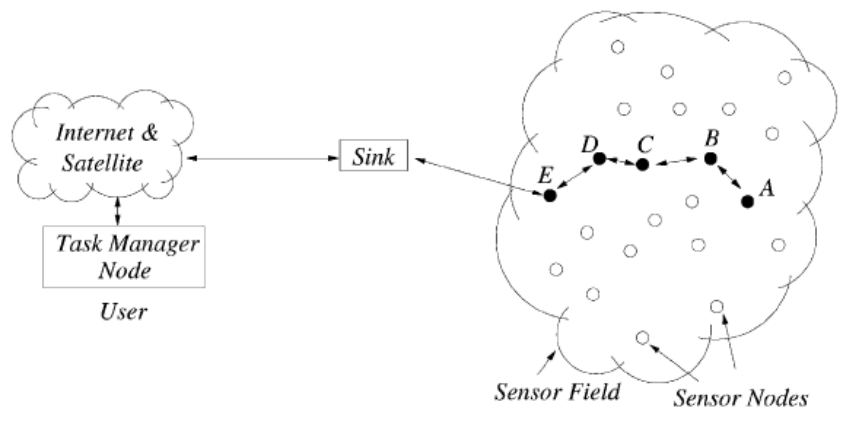

Figure 2. Sensor nodes communicate in a sensor field
A WSN can be considered at a higher level as the combination of two different network entities [4]: the data acquisition network and the data dissemination network. The data acquisition network is a collection of sensor nodes and the base station. The sensor nodes measure physical data while the base station collects the information from the nodes and sends control data to the network environment. The data dissemination network interfaces the data acquisition network to a user and is a collection of wired and wireless networks.

In order to ensure communication quality between nodes, many protocols have been proposed to address the single or multiple hop change in WSN communication. With the industry development of the IoT, a protocol that can integrate services easily is being promoted, in which the SOA and the semantic web services can play an important role.

\section{EWSN FRAMEWORK}

RFID is another important technology of the IoT. EPC is the electronic product code. The RFID technology assigns a unique ID EPC which maps to a real-world object to a RFID tag. [11]

In order to build a network out of these objects so that a unique EPC can easily present the information about those objects, a seamless global network of physical objects is constructed and the EPC network is an open standard of this architecture.

While the EPC uniquely identifies objects, the EPC Network contains the information about it. And the Physical Markup language defines a standardized generic markup language for information interchange modeling and encapsulation of the data captured by the RFID readers. The Object Name Service provides URLS to authoritative information relevant to an object [11].

Since the WSN and RFID technologies are not actually separated from each other in the IoT, more and more applications integrate the WSN and RFID to bring much more advantages in business. Therefore, we use the EPC network as a framework of the integrated system, which can process both WSN and RFID data and information. This extended system is defined as EWSN, both RFID tags and WSN sensors in the architecture are called EWSN nodes [12]. Figure 3 gives the architecture of EWSN.

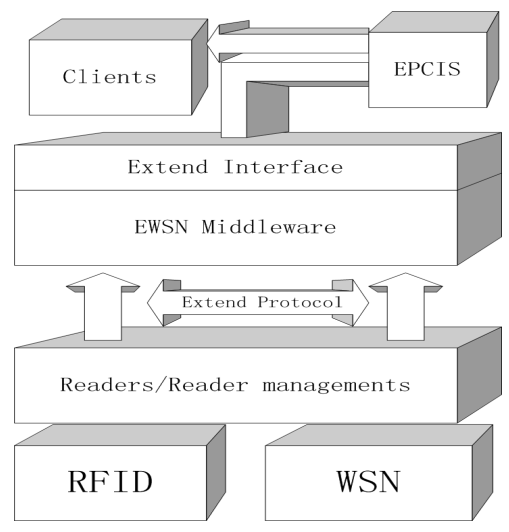

Figure 3. The architecture of EWSN 
EWSN can gather, filter and transfer the data and information of the EWSN nodes. In order to achieve this, a EWSN middleware system is designed to collect and distribute data [3-4] [10]. The EWSN middleware contains two kinds of event objects: WSN event object and RFID event object, and a rule is defined to monitor the event and data process, which contains: events, conditions, and actions [8]. In addition, the lifetime of the sensor data is extended by defining a duration variable in the middleware system because the content differences between RFID and sensor events lead to differences in the event granularity [10].

\section{SERVICE-CENTRIC EWSN}

Because EWSN cannot deal with the dynamic and heterogeneous integrated system well, we apply Semantic Web Service to the EWSN system to build a flexible system with automatic service composition which can describe, discover services from different EWSN nodes and networks using XML and SOAP standards [5]. We define the service-centric EWSN as SWSN, where both of the RFID tags and the WSN sensor nodes are intelligent SWSN nodes.

Web services are proposed to provide interoperability between diverse applications [11]. Web service and the independent interfaces make the integration of heterogeneous systems easier [1]. The UDDI (Discover and Integration), WSDL (Web Service Description Language), SOAP (Simple Object Access Protocol) languages define standards for service discovery, description and so on [3] [11]. Figure 4 is a typical architecture of Web Service.

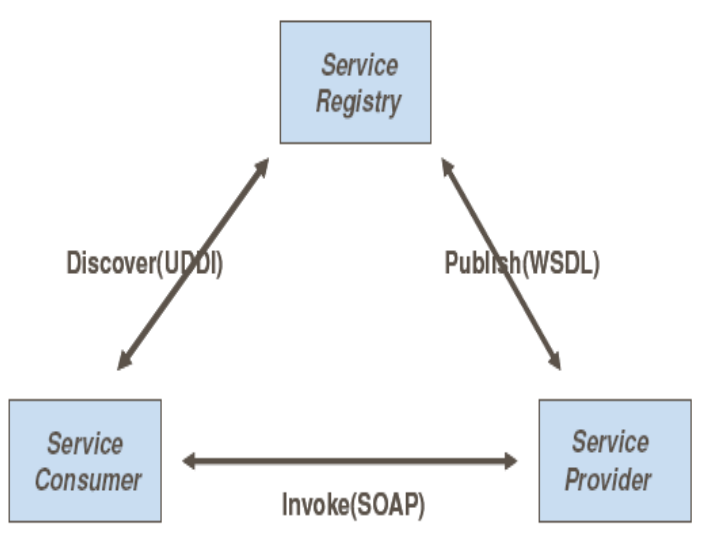

Figure 4. Typical architecture of Web Service.

There are several approaches for services composition such as BPEL and the Semantic Web is designed to deal with the dynamic composition of existing services [5]. Semantic Web Services can automatically discover, composite, invoke and monitor the Web services by providing an ontology description In addition, we can describe the services in semantic annotations for WSDL and XML schema, or provide a ontology written in the ontology language OWL (Web Ontology Language) [1]. Figure 5 gives the SWSN service architecture model [3] [13].

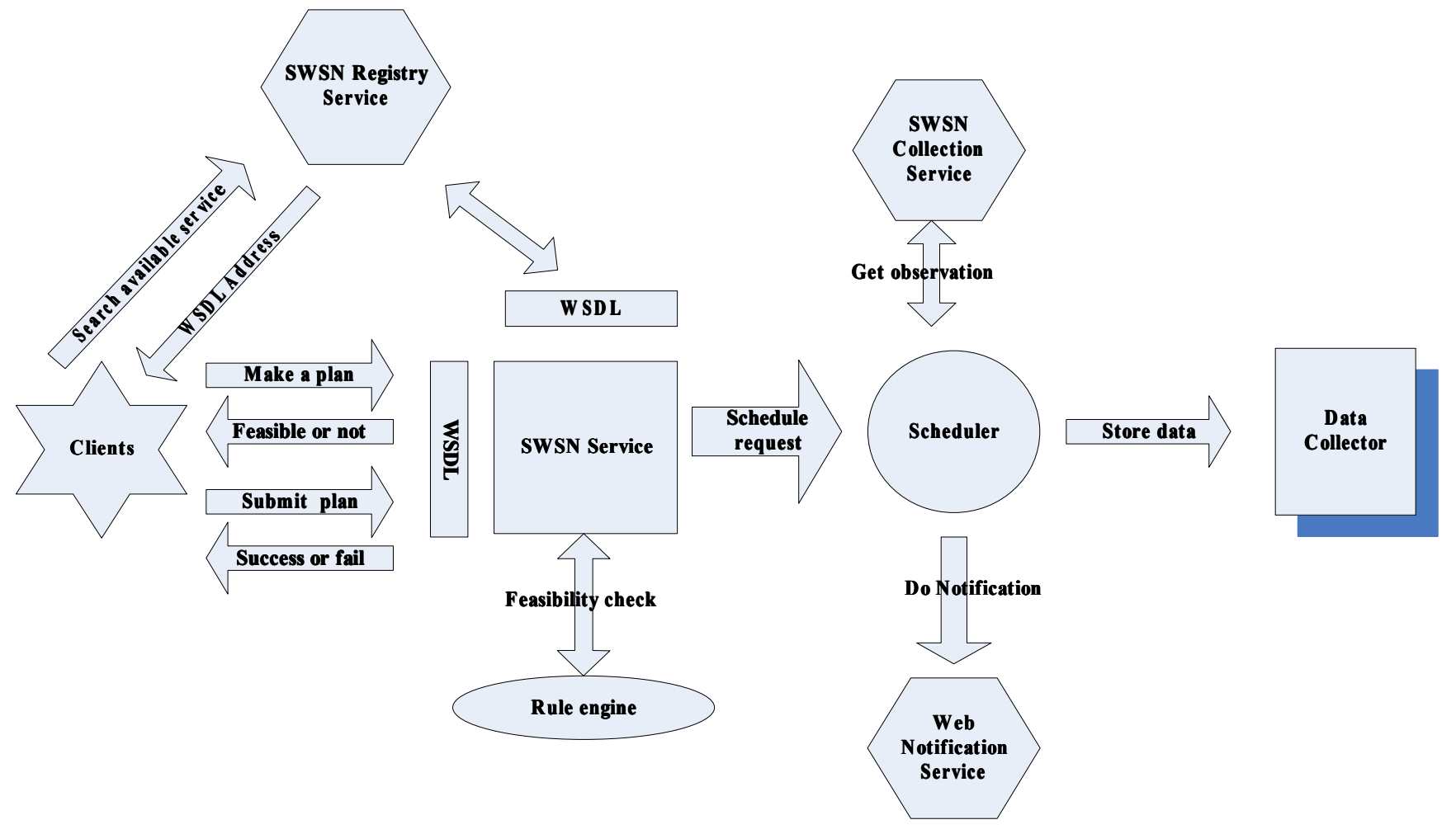

Figure 5. Service architecture model of SWSN. 
We use the service-oriented property to describe the sensing data with ontology [3]. It can share the common understanding of the SWSN information structure among
SWSN nodes. It can enable reuse and separate and analyze the data and information of the nodes as services. Figure 6 gives the taxonomy of the possible ontology design [5].

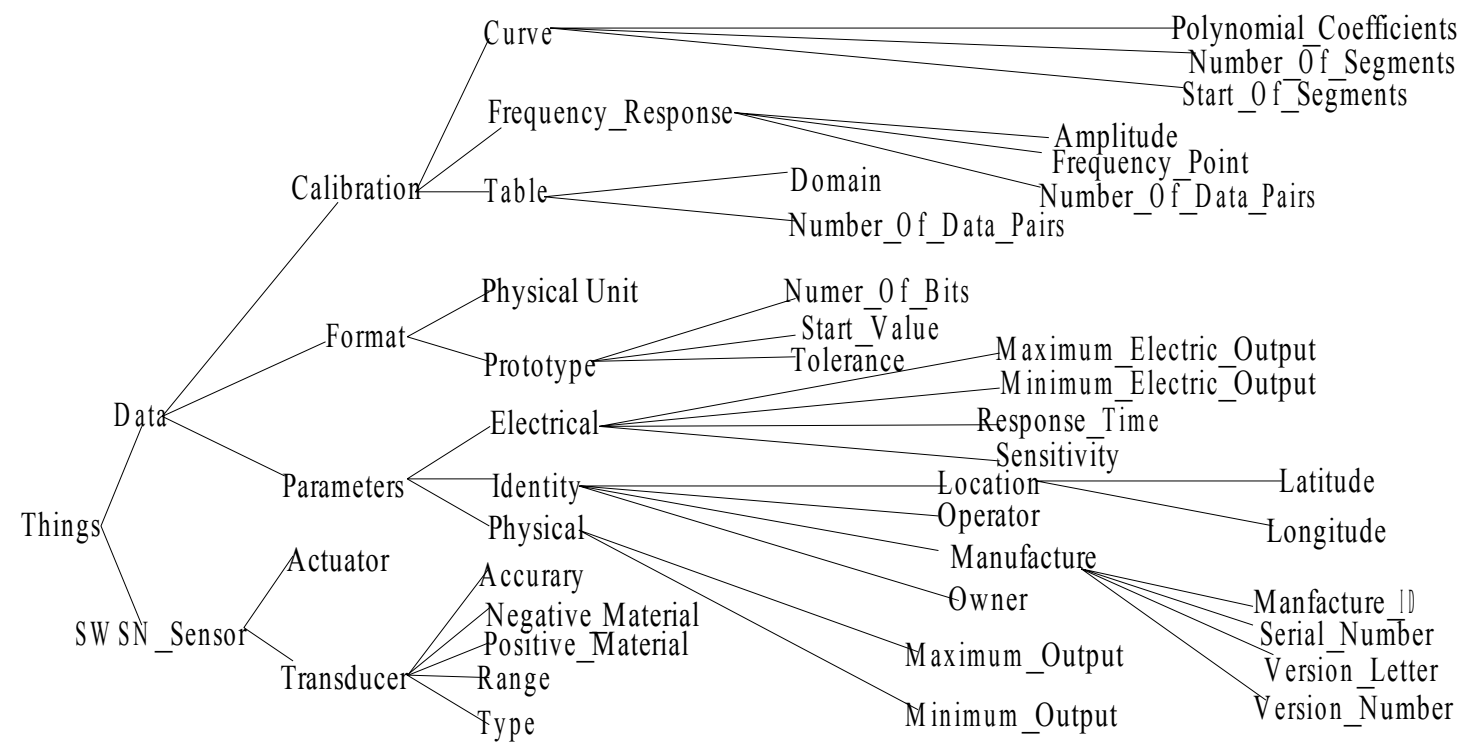

Figure 6. Taxonomy of the possible ontology design.

In the architecture of SWSN, the SWSN nodes and the information gathered by the nodes are distributed reusable resources in the real world. In this service-centric environment, each SWSN node is a service provider and a consumer, who are dynamic. Figure 7 shows the implementation architecture of a real-world application of SWSN.

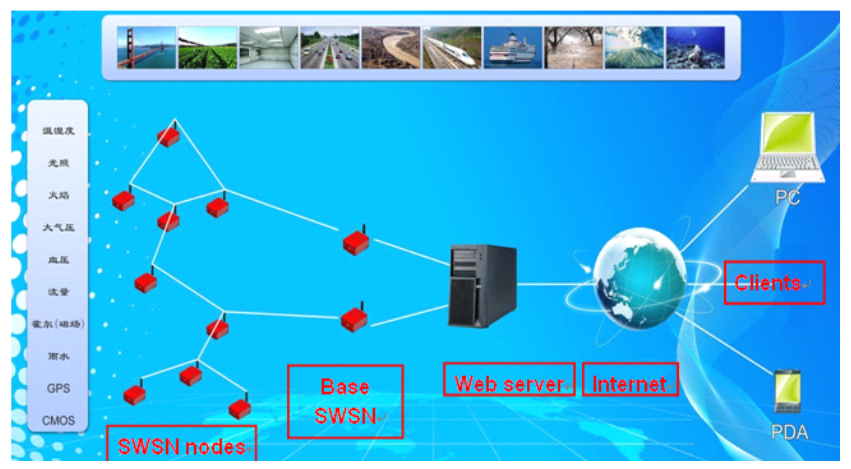

Figure 7. The reusable resources gathered by SWSN.

\section{IMPLEMENTATION AND CHALLENGES}

\section{A. SWSN Applications}

SWSN is a part of the Intelligent Internet of Things (IIoT) project in the lab of SINOIOTS.

We have implemented a lot of applications in industry, such as health care, smart home, environment protection, and so on. We believe that the future of the IoT is the Internet of Service which is based on the developments in the IoT industry. Figure 8 shows the interface platform of these applications.

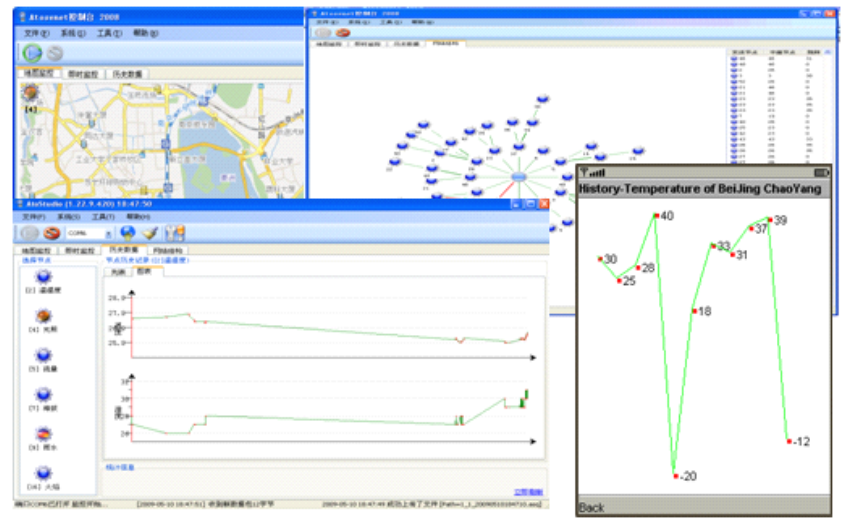

Figure 8. Interface platform of SWSN applications

\section{B. Challenges in SWSN}

Since SWSN applications are interacted with the real world environment, we should pay more attention to the quality of service of SWSN. While SWSN inherit most of the QoS challenges from general wireless networks, their particular characteristics pose some unique challenges [6]. The constraints on resources such as energy severely limit the nodes for remote or inhospitable environment applications. In SWSN, traffic flows from a larger number of sensor nodes to a small subset of sink nodes may lead to a traffic congestion problem [6]. Other problems include data redundancy, network 
dynamics, energy balancing, and packer criticality, which are also big challenges in SWSN.

\section{CONCLUSION}

In this paper, we introduced the use of the EPC network to integrate the RFID and WSN system with Semantic Web Service. An integrated architecture of RFID and WSN extended from the EPC network architecture was defined as EWSN. The service-centric SWSN was presented and the implementation challenges were discussed. The integration of WSN and RFID provide many more advantages in the realworld pervasive computing and the extension of the EPC architecture with SOA can provide a smooth way to integrate the RFID and WSN system for better IoT applications.

\section{ACKNOWLEDGMENT}

We would like to thank the IoT research laboratory of Sinoiots for all the support provided to this work.

\section{REFERENCES}

[1] B. Medjahed, A. Bouguettaya, and A. Elmagarmid, "Composing Web Services on the Semantic Web," The VLDB J, vol. 12, no. 4, June 2003, pp. 333-351.

[2] M. Chen and T. Kwon, "Data dissemination based on mobile agent in wireless sensor Networks," Proceedings of the IEEE Conference on Local Computer Networks, Sydney, Australia, November 2006, pp. 6382.

[3] X. Chu and R. Buyya, "Service oriented sensor web." Mahalik, N. P. (ed), Sensor Network and Configuration: Fundamentals, Standards, Platforms, and Applications. Springer-Verlag, ISBN: 978-3-540-373643, Germany, Jan. 2007, pp.51-74.

[4] F.C. Delicato, P.F. Pires, L. Pirmez, and L. Rust, "A flexible middleware system for wireless sensor networks," Proceedings of the ACM/IFIP/USENIX International Middleware Conference, Rio de Janeiro, Brazil, July 2003, pp. 21-45.

[5] J. H. Kim, H. Kwon, D. H. Kim, H. Y. Kwak, and S. J. Lee, "Building a service-oriented ontology for wireless sensor networks," Computer and Information Science, 2008. ICIS 08. Seventh IEEE/ACIS International Conference on, May 2008, pp. 649-654.

[6] D. Chen and P. K. Varshney, "QoS support in wireless sensor networks: A survey," Proceedings of the 2004 international confeference on Wireless Networks, Las Vegas, Nevada, USA June 2004, pp. 227-233.

[7] T. S. Lopez and D. Kim, "Wireless sensor networks and RFID integration for context aware services," Technical report, Auto-ID Labs, March 2008.

[8] M. Palaniswami, G. Egan Sophia Kaplantzis, N. Mani, "Security models for wireless sensor networks," Technical report, University of Texas at Arlington, May 2004.

[9] I.F. Akyildiz, W. Su, Y. Sankarasubramaniam, and E. Cayirci, "Wireless sensor networks: A survey," Computer Networks, April 2002, pp. 393422.

[10] W. Wang, J. Sung, and D. Kim. "Complex event processing in epc sensor network middleware for both rfid and wsn," Proceedings of the 11th IEEE Symp. On Object Oriented Real-Time Distributed Computing (ISORC), May 2008, pp. 165-169.

[11] E. Sirin, J. Hendler, B. Parsia, "Semi-automatic composition of web services using semantic descriptions," Web Services: Modeling, Architecture and Infrastructure Workshop in Conjunction with ICEIS, Berlin, Germany,June 2003.

[12] W. Heinzelman, A. Murphy, H. Carvalho, and M. Perillo, "Middleware to support sensor network applications," IEEE Network Magazine, vol 10, no. 2 July 2004, pp. 6-14.
[13] F. Patrik, "Building a smart hospital using RFID technologies," Proceedings of the 1st European Conference on eHealth (ECEH06), Fribourg, Switzerland, Oct. 2006, pp. 12-23. 